\title{
A Continuum Analogue of the Lattice Gas
}

\author{
R. F. Streater \\ Mathematics Department, Bedford College, Regents Park \\ London, N.W. 1.
}

Received November 25, 1968

\begin{abstract}
We construct a Hilbert space $\mathscr{H}$, spanned by vectors $|\mathcal{O}\rangle$, where $\mathcal{O}$ is a bounded measurable set in $\mathbb{R}^{v}(v=$ dimension of space), and interpret $|\mathcal{O}\rangle$ as a state where all points $x \in \mathcal{O}$ are occupied by an incompressible fluid, and $x \notin \mathcal{O}$ unoccupied. $\mathscr{H}$ is generated by applying unitary "filling operators" $U(\mathcal{O})$ to a cyclic vector $|\phi\rangle$, the completely unoccupied state. The operators $U(\mathcal{O})$ generate a commutative $c^{*}$-algebra, of which the hermitian elements are interpreted as the observables of the theory.

All the $\infty$-divisible representations of the symmetric group of order 2 are found. We give a generalization to a theory with any number of particle types.
\end{abstract}

\section{A Continuum Analogue of the Lattice Gas}

A configuration of a lattice gas is defined, in one dimension, by a sequence of noughts and ones. More generally, [1], in $v$ dimensions, if there are $n$ types of particle, a configuration is given by a map from $Z^{v}$ to $(0,1, \ldots, n)$. In this paper we show how to realize the configuration space of a system describing a gas in a continuum, where it is assumed that each point is either occupied or unoccupied.

\section{Continuous Tensor Products}

In their general analysis of complete Boolean algebras of factors, ARAKI and Woods [2] define a continuous tensor product of a family of Hilbert spaces $\mathscr{H}_{x}$ relative to a vector field $\Omega_{x}$, to be $\exp \int_{\oplus}\left(\mathscr{H}_{x} \ominus \Omega_{x}\right) d x$, that is, the Fock space over the direct integral of the spaces perpendicular to $\Omega_{x}$. Their analysis shows that any complete Boolean algebra of factors can be embedded in such a space. GuICHARDET [3] has given a definition of continuous tensor products of Banach spaces, and a tentative definition of products of Hilbert spaces. There is no obvious reason why the scalar product he defines on the tensor pioduct should be positive-definite in general. But he is able to prove positive definiteness for an example, in which the base-space is compact, by using the Fock space idea. Independently, D. DuBIN and the author [4] working from physical considerations, arrived at a model with indefinite metric, indicating that continuous tensor products do not always exist. Further examples 
satisfying the positivity were given in [5-9]. The connection between the various formalisms is given by a suitable modification [8] of the Araki-Woods embedding theorem: any continuous tensor product in the sense of [3] or [5] can be embedded in a Fock space (see also [9]).

In this paper the analogue of this for finite groups is given. The resulting local theory may be regarded as the continuum version of the classical lattice gas. For the symmetric group $S_{2}$, all infinitely divisible representations are found.

Let $G$ be a finite group with the Borel structure induced by the discrete topology; let $e$ denote the identity element of $G$. Let $U$ be a cyclic unitary representation of $G$ in a Hilbert space $\mathscr{H}$, with cyclic vector $\omega$. Let $\mathscr{M}\left(\mathbb{R}^{v}, G\right)$ be the set of measurable functions $f$ from $\mathbb{R}^{v}$ to $G(v=1,2$ or 3 say), such that to each $f$ there exists a compact set $K \subset \mathbb{R}^{v}$ such that $f(x)=e$ if $x$ is outside $K$. The smallest compact set with this property is called the support of $f$; it is always a measurable set. $\mathscr{M}\left(\mathbb{R}^{v}, G\right)$ becomes a group under pointwise multiplication: $f g(x)$ $=f(x) g(x)$. The set of $x$ such that $f(x)$ takes a given value in $G$ is a measurable set, so the function $(\omega, U(f(x)) \omega)$ is a measurable function. Suppose that $(\omega, U(g) \omega) \neq 0$ for all $g$, and choose a branch of $\log (\omega, U(f(x)) \omega)$, for each $f \in \mathscr{M}\left(\mathbb{R}^{v}, G\right)$, such that

$$
\log \left(\omega, U\left(f^{-1}(x) g(x)\right) \omega\right)=0
$$

if $x \notin \operatorname{supp} f \cup \operatorname{supp} g$. We furnish $\left[\mathscr{M}\left(\mathbb{R}^{v}, G\right)\right]$ (the free complex vector space generated by the set $\mathscr{M}\left(\mathbb{R}^{v}, G\right)$ ) with the sesquilinear form, defined on $\mathscr{M}\left(\mathbb{R}^{v}, G\right)$, by

$$
\langle f, g\rangle=\exp \int d x \log \left(\omega, U\left(f^{-1} g(x)\right) \omega\right)
$$

and from there by linearity in $g$ and anti-linearity in $f$. Since the support of $f^{-1} g$ is compact, and $U(e)=1$, the integral in (1) is always over a compact region.

We say the tensor product $\bigotimes_{x} U$ exists (for a particular definition of logarithm) if $(1)$ is positive semi-definite. In this case, $\langle$,$\rangle defines a$ canonical Hilbert space, denoted $\bigotimes_{x} \mathscr{H}$, by the separation and completion of $\left[\mathscr{M}\left(\mathbb{R}^{v}, G\right)\right]$ with respect to the semi-norm defined by $\langle$,$\rangle . We$ denote by $\Omega=\bigotimes_{x} \omega$, or $|\emptyset\rangle$, the vector corresponding to the identity $E$ of $\mathscr{M}\left(\mathbb{R}^{v}, G\right) ; E$ is the map $E(x)=e \in G$ for all $x \in \mathbb{R}^{v}$. One gets a representation of the group $\mathscr{M}\left(\mathbb{R}^{v}, G\right)$ by unitary operators on $\bigotimes_{x} \mathscr{H}$ as follows (the analogue of the construction of representations of the current algebra, see [5]). For each fixed $f$, the group multiplication $\psi \rightarrow \psi f$ in $\mathscr{M}\left(\mathbb{R}^{v}, G\right)$ is a scalar-product preserving map of $\mathscr{M}\left(\mathbb{R}^{v}, G\right)$ to $\mathscr{M}\left(\mathbb{R}^{v}, G\right)$. It can therefore be extended to a unitary operator $\pi(f)$ on 
$\bigotimes_{x} \mathscr{H}$, i.e., a map $\pi: \mathscr{M}\left(\mathbb{R}^{v}, G\right) \rightarrow \operatorname{Aut}\left(\bigotimes_{x} \mathscr{H}\right)$. We can define the local $c^{*}$-algebras of the theory as follows: let $\mathcal{O} \subset \mathbb{R}^{v}$ be a bounded open set; the set of operators $\{\pi(f)$; supp $f \subset \mathcal{O}\}$ generate a $c^{*}$-algebra denoted $\mathscr{A}(\mathcal{O})$; the quasi-local algebra $\mathscr{A}$ is defined to be the $c^{*}$-algebra generated by all the $\mathscr{A}(\mathcal{O})$. If $\mathcal{O}_{1} \subset \mathbb{R}^{v}$, we define $\mathscr{A}\left(\mathcal{O}_{1}\right)$ to be the $c^{*}$-algebra generated by $\mathscr{A}(\mathcal{O}), \mathcal{O} \subset \mathcal{O}_{1}$.

The von Neumann algebras $\mathscr{A}(\mathcal{O})^{\prime \prime}=\mathscr{R}(\mathcal{O})$ generated by the $\mathscr{A}(\mathcal{O})$ give rise to a structure very similar to that studied by ARAkI and Woops [2], except that in general, they are not factors, and that the so-called "duality theorem", $\mathscr{R}(\mathcal{O})^{\prime}=\mathscr{R}\left(\mathbb{R}^{v}-\mathcal{O}\right)$, does not necessarily hold. To see this, consider $G=S_{2}$. Then $\mathscr{M}\left(\mathbb{R}^{v}, G\right)$ is abelian and so is each $\mathscr{R}(\mathcal{O})$. This means that $\mathscr{R}(\mathcal{O})$ are not factors. The fact that $\mathscr{R}(\mathcal{O})^{\prime}$ $\neq \mathscr{R}\left(\mathbb{R}^{v}-\mathcal{O}\right)$ is also easy to see. In spite of this, an embedding theorem, similar to that of ARAKI and Woods, can be demonstrated (see [8]).

If $U(g) \omega$ takes $n+1$ distinct values as $g$ runs over $G$, we may interpret $\bigotimes_{x} \mathscr{H}$, or rather the total set $\left\{\pi(f)|\emptyset\rangle ; f \in \mathscr{M}\left(\mathbb{R}^{v}, G\right)\right\}$ as the space of configurations, in which each point can be in one of $n+1$ states; $\omega$ denotes the empty state and the other $n$ vectors could denote $n$ different particle types; the vector $|\emptyset\rangle$ denotes the state of no particles; it is a cyclic vector for $\mathscr{A}$.

\section{Infinitely Divisible Group Representations}

Definition. We say a group representation $U$, with cyclic vector $\omega \in \mathscr{H}$, is infinitely divisible if, to any positive integer $q$, there is a representation, denoted $U^{1 / q}$, on a Hilbert space $\mathscr{H}^{1 / q}$ with cyclic vector $\omega^{1 / q}$, such that $U$ is equivalent to $U^{1 / q} \underbrace{q \text { factors }}_{\otimes \cdots \otimes} U^{1 / q}$ (as a representation of $G$, the diagonal subgroup of $G \times \underbrace{q \text { factors }}_{G \times \cdots \times G)}$ restricted to the cyclic subspace generated from $\omega^{1 / q} \otimes \cdots \otimes \omega^{1 / q}$ by the action of the group. In this equivalence, $\omega$ and $\omega^{1 / q} \otimes \cdots \otimes \omega^{1 / q}$ are to correspond. This concept was introduced in [8]; the point is the following theorem.

Theorem 1. There exists a choice of logarithm making (1) positive semidefinite if and only if $U$ is infinitely divisible.

Proof. If $U$ is infinitely divisible, and $q$ is given, we may write

$$
(\omega, U(g) \omega)=\left(\omega^{1 / q}, U^{1 / q}(g) \omega^{1 / q}\right)^{q}
$$

where $\left.f, g \rightarrow(\omega)^{1 / q}, U^{1 / q}\left(f^{-1} g\right) \omega^{1 / q}\right)$ is a semi-definite form on $G$. We may therefore define a branch of the $q^{\prime}$ th root of $(\omega, U(g) \omega)$ by $(\omega, U(g) \omega)^{1 / q}$ $=\left(\omega^{1 / q}, U^{1 / q}(g) \omega^{1 / q}\right)$, and still get a positive semi-definite form on $G$. If $r=p / q$ is any rational, raising this equation to the power $p$ shows that $(\omega, U(g) \omega)^{r}$ is positive semi-definite, and by continuity, $(\omega, U(g) \omega)^{\tau}$, 
$\tau>0$, may be defined so that it is positive semi-definite, i.e.,

$$
\sum_{i, j}^{N} \bar{\alpha}_{i} \alpha_{j}\left(\omega, U\left(g_{i}^{-1} g_{j}\right) \omega\right)^{\tau} \geqq 0 .
$$

Now consider the bilinear form (1) on $\mathscr{M}\left(\mathbb{R}^{p}, G\right)$. The function $\left(\omega, U\left(g_{i}^{-1} g_{j}(x)\right) \omega\right)$ takes only a finite number of values for each $i, j$, and the set of $x$, such that it takes a given value, is measurable. Thus for fixed $i, j, \mathbb{R}^{v}$ is divided into a finite number of measurable sets, at most $n$, on each of which $\left(\omega, U\left(g_{i}^{-1} g_{j}(x)\right) \omega\right)$ is constant. Thus, as $i=1,2, \ldots N$ and $j=1,2, \ldots N$, we may split $\mathbb{R}^{v}$ into at most $n^{N^{2}}$ measurable sets $V_{\lambda}$ say. We pick a point $x_{\lambda} \in V_{\lambda}$, and choose the branch of the logarithm in (1) so that

$$
\begin{aligned}
\left\langle f_{i}, f_{j}\right\rangle & =\prod_{\lambda} \exp \int_{V_{\lambda}} d x \log \left(\omega\left(f_{i}^{-1}\left(x_{\lambda}\right) f_{j}\left(x_{\lambda}\right)\right) \omega\right) \\
& =\prod_{\lambda}\left(\omega, U\left(f_{i}^{-1}\left(x_{\lambda}\right) f_{j}\left(x_{\lambda}\right)\right) \omega\right)^{\tau_{\lambda}}
\end{aligned}
$$

(where $\tau_{\lambda}$ is the volume of $V_{\lambda}$ ). For each $\lambda,\left(\omega, U\left(f_{i}^{-1}\left(x_{\lambda}\right) f_{j}\left(x_{j}\right)\right) \omega\right)^{\tau_{\lambda}}$ is positive semi-definite, so the product over $\lambda$ is too. This proves that $\otimes U$ exists.

Conversely, if there is a continuous tensor product, we may choose, in particular, elements $g(x)$ of $\mathscr{M}\left(\mathbb{R}^{v}, G\right)$ such that $g(x)$ is constant on $\mathcal{O} \subset \mathbb{R}^{v}$, and equal to $e$ outside $\mathcal{O}$. This leads to the positive definiteness of the form $\exp _{x} \int_{\mathcal{O}} d x \log (\omega, U(g) \omega)=(\omega, U(g) \omega)^{\tau(\mathcal{O})}$ where $\tau(\mathcal{O})$ is the volume of $\mathcal{O}$; here the branch of $(\omega, U(g) \omega)^{\tau}$ is determined by the choice of branch of logarithm which, by assumption, may be chosen to make it positive definite. Choosing $\mathcal{O}$ such that $\tau(\mathcal{O})=1 / q$, we see that $(\omega, U(g) \omega)^{1 / q}$ defines a positive semi-definite function, so

$$
f, g \rightarrow\left(\omega, U\left(f^{-1} g\right) \omega\right)^{1 / q}
$$

defines an invariant sesqui-linear form on $G$, and thus, by a well known construction, it defines a cyclic representation of $G$, say $U^{1 / q}$ with cyclic vector $\omega^{1 / q}$, such that

$$
(\omega, U(g) \omega)=\left(\omega^{1 / q}, U^{1 / q}(g) \omega^{1 / q}\right) .
$$

This proves that $U$ is $\infty$-divisible.

\section{The Embedding Theorem}

It is clear that $U, \omega$ is infinitely divisible if and only if $(\omega, U(g) \omega)^{\tau}$ is a positive semi-definite function on $G$ for all $\tau>0$. If in addition it never vanishes we may define $F(g)=\log (\omega, U(g) \omega)$, so $e^{F^{\prime}(g)}$ is a positive semi-definite function on $G$. When $G=\mathbb{R}$ this result is well-known; see 
Gelfand and Vilenkin, Generalized Functions, Vol. IV, Theorem 3, p. 278. In the same way as Theorem 4, p. 279 , one can prove that $e^{F^{\prime}(g)}$ is positive semi-definite if and only if $F(g)$ is a conditionally positive function on $G$, i.e., $\sum_{j=1}^{n} \sum_{k=1}^{n} F\left(g_{j}^{-1} g_{k}\right) \bar{\alpha}_{j} \alpha_{k} \geqq 0$ for all $g_{j} \in G$ and $\alpha_{k} \in \mathbb{C}$, such that $\sum \alpha_{i}=0$. The following theorem is proved in [8]: A function $F(g)$ is conditionally positive if and only if the form

$$
\langle g, h\rangle=F\left(g^{-1} h\right)-F\left(g^{-1}\right)-F(h)
$$

defines by linear extension a positive semi-definite form on $[G]$. (For $G=\mathbb{R}$, this is proved in [11].)

Now let $U$ be $\infty$ divisible on a Hilbert space $\mathscr{H}$, with cyclic vector $\omega$, and $F(g)=\log (\omega, U(g) \omega)$. Let $\mathscr{K}$ be the separated completed space of $[G]$ furnished with the form (2). Clearly, $\langle e, e\rangle=0$, so $e$ represents the zero of $\mathscr{K}$. If $G_{0} \subset G$ is the subgroup such that $U(g) \omega=e^{i \lambda} \omega$, then $\langle$,$\rangle is in fact a semi-definite form on G / G_{0}$, which may thus be regarded as a total set in $\mathscr{K}$. The set of elements $\left\{\exp q / q \in G / G_{0}\right\}$ is not total in $\exp \mathscr{K}$, however, since $G / G_{0}$ has a finite number of elements and $\exp \mathscr{K}$ is infinite dimensional unless $\mathscr{K}$ is zero. Let $\mathscr{L} \subset \exp \mathscr{K}$ be the subspace generated by the elements $\exp q$. We are then able to identify $\mathscr{H}$ with $\mathscr{L}$. More exactly, we define the following map $V$ from $\mathscr{H}$ to $\exp \mathscr{K}$ :

$$
V(U(g) \omega)=(\omega, U(g) \omega) \exp \{g\}
$$

where $\{g\}$ is the equivalence class in $G / G_{0}$ containing $g$.

Theorem 2. $V$ can be extended to a unitary map from $\mathscr{H}$ to $\mathscr{L}$ (see [8]).

This is the main embedding theorem; it shows that any $\infty$-divisible representation can be embedded in a suitable Fock space.

In the same way, the continuous tensor product $\bigotimes_{x} \mathscr{H}$ can be embedded in the Fock space over $\int_{\oplus} d x \mathscr{K}$. For, the vector $U(f)(\otimes \omega)$ may be represented by the element $f \in \mathscr{M}\left(\mathbb{R}^{v}, G\right)$, and such an $f$ may be regarded as an element of $\int_{\oplus} \mathscr{K} d x$; for $f(x)=e$ outside a compact set, and $e$ represents the zero element of $\mathscr{K}$. Thus $f(x)$ defines a squareintegrable vector field in $\mathscr{K}$. Let $\hat{f}$ denote this element of $\int_{\oplus} \mathscr{K} d x$. Define a map $W: \mathscr{M}\left(\mathbb{R}^{v}, G\right) \rightarrow \exp \int_{\oplus} \mathscr{K} d x$ by $W(f)=\left\{\exp \int d x \log (\omega, U(f(x)) \omega)\right\}$ $\exp \hat{f}$. The map $W$ is a scalar-product preserving map from $\mathscr{M}\left(\mathbb{R}^{v}, G\right)$, furnished with the scalar product (1), to $\exp \int_{\oplus} \mathscr{K} d x$; it therefore defines a unitary map from $\bigotimes_{x} \mathscr{H}$ to $\exp \int_{\oplus} \mathscr{K} d x$. This is our version of the Araki-Woods embedding theorem. In our case there is no obvious reason why the map should be onto. 


\section{Some Examples}

Let $G$ be a finite group of order $N$, and furnish $[G]$ with the bilinear form

$$
\left(g_{i}, g_{j}\right)=\delta_{i j}+\left(1-\delta_{i j}\right) \cos \theta \text {. }
$$

This is positive definite if $0<\theta \leqq \pi / 2$, and never vanishes if $0<\theta<\pi / 2$. Furnished with this form, $[G]$ becomes a Hilbert space of dimension $N$. Since (4) is invariant under the left action of $G$ on $G$, the map $g_{i} \longmapsto g g_{i}$ induces a unitary representation $U(g)$ of $G$ on $[G]$, which is equivalent to the regular representation (since the ideal structure does not depend on any particular bilinear forms having been chosen). Any $g \in G$ is a cyclic vector, say $\omega$, of the regular representation.

The matrix $\left(g_{i}, g_{j}\right)^{1 / q}$ (real branch chosen) is also positive definite on $[G]$, since $1^{1 / q}=1$ and $(\cos \theta)^{1 / q}=\cos \alpha$ for some real $\alpha$. Therefore for each $\theta<\pi / 2$ the representation $(U, \omega)$ is $\infty$-divisible, and $(\omega, U(g) \omega)$ $\neq 0, g \in G$.

The symmetric $S_{n}$ possesses many $\propto$-divisible representations of dimension $n$. For, if $|j\rangle, j=1, \ldots, n$ denote $n$ symbols, with scalar product

$$
(i, j)=\delta_{i j}+\left(1-\delta_{i j}\right) \cos \theta
$$

we may define a unitary representation of $S_{n}$ by

$$
U(\pi)|i\rangle=|\pi(i)\rangle \text { for all } \pi \in S_{n} .
$$

It is worthwhile computing the scalar product (2) which defines the space $\mathscr{K}$ of the embedding theorem, for these two examples. We find: $\langle e, e\rangle=\langle e, g\rangle=0 ;\langle g, h\rangle=-\log \cos \theta g \neq g ;\langle g, g\rangle=-2 \log \cos \theta$, $g \neq e$. This is a positive semi-definite form, as required by (2). In a similar way, one verifies that the form $\langle$,$\rangle in the second example, is$ positive semi-definite on $S_{n}$.

In the particular case $G=S_{2}$, these two representations coincide. In fact, we can easily find all infinitely divisible representations of $S_{2}$. Any cyclic representation has dimension $\leqq 2$ and is defined by a form (,) on $G$, invariant under left multiplication. The identity representation is always $\infty$-divisible. The necessary and sufficient condition on the form (,) is that (2) be positive semi-definite. If $g \neq e$, we have $\langle g, e\rangle=0$ and

$$
\langle g, g\rangle=-\log \left(g^{-1}, e\right)-\log (g, e)=-2 \log (g, e) \geqq 0 .
$$

Therefore, (,) leads to an $\infty$-divisible representation if and only if $(g, e)$ is real and $0 \leqq(g, e) \leqq 1$. These are just those forms discussed above, with $(e, g)=\cos \theta$. In this example, we may label a total set of vectors in $\otimes \mathscr{H}$ by a bounded measurable set $E$ of occupied states, with

with

$$
\left(E_{1}, E_{2}\right)=\exp \int d x \log \left(g_{1}(x), g_{2}(x)\right),
$$

$$
\begin{aligned}
g_{j}(x) & =e, \quad x \in E_{j} \\
& \neq e \text { otherwise } .
\end{aligned}
$$


Then

$$
\begin{aligned}
\left(g_{1}(x), g_{2}(x)\right) & =\cos \theta, \quad x \in E_{1} \cup E_{2}-E_{1} \cap E_{2} \\
& =1 \text { otherwise. }
\end{aligned}
$$

This gives the scalar product

$$
\left(E_{1}, E_{2}\right)=(\cos \theta)^{\tau\left(E_{1} \cup E_{2}-E_{1} \cap E_{2}\right)} .
$$

Our analysis guarantees that this furnishes the linear space generated by measurable sets with a positive semi-definite form. The corresponding Hilbert space is separable; for there exists a countable set of measurable sets $E_{i}$ such that given $E$ and $\varepsilon>0$, there exists an $i$ such that $E_{i}$ approximates $E: \tau\left(E-E_{i}\right)<\varepsilon$ and $\tau\left(E_{i}-E\right)<\varepsilon$. The corresponding set of vectors $\left|E_{i}\right\rangle$ form a total set.

The translation operator $T_{a}$ in $\mathbb{R}^{v}$ acts in a natural way on $\mathscr{M}\left(\mathbb{R}^{v}, G\right): T_{a} f(x)=f(x+a)$. The scalar product (1) is invariant under this action, so each $T_{a}$ defines a unitary operator in $\bigotimes \mathscr{H}$; the $T_{a}$ realize the group $\mathbb{R}^{v}$. The action of $T_{a}$ is continuous on $\bigotimes \mathscr{H}$. The unitary operators $U(E)$ defined by $U(E)\left|E^{\mathrm{I}}\right\rangle=\left|E \cup E^{\mathrm{I}}-E \cap E^{\mathrm{I}}\right\rangle$ generate a commutative quasi-local algebra as $E$ runs over bounded sets. The cluster property, $\left\langle E, T_{a} E^{\mathrm{I}}\right\rangle \rightarrow\langle E \mid \emptyset\rangle\left\langle\emptyset \mid E^{\mathrm{I}}\right\rangle$ as $|a| \rightarrow \infty$, also holds. We thus have all the ingredients of a continuum theory of a gas with a maximum density. Similarly, a continuous product of $S_{n+1}$ leads to a gas of $n$ different particles. The $c^{*}$-algebras are not commutative in that case, but are obviously asymptotically abelian.

\section{References}

1. Ruelle, D.: J. Math. Phys. 8, 1657 (1967).

2. Arakr, H., and E. J. Woods: Publ. Research Inst. Math. Sci., Kyoto, Ser. A, Vol. 2, 157 (1966).

3. Guichardet, A.: Commun. Math. Phys. 5, 262 (1967).

4. Dubin, D., and R. F. Streater: Nuovo Cimento 50, 154 (1967).

5. Streater, R. F.: Nuovo Cimento อ̃3, A 487 (1968).

6. - Lectures at Karapcz (Poland) Winter School, February 1968.

7. - , and A. Wulfsohn: Nuovo Cimento 57 B, 330 (1968).

8. - Lectures at Varenna Summer School, 1968.

9. Araki, H.: Facterizable Representations of Current Algebra. Preprint.

10. Guichardet, A.: Algèbres d'Observables Associées aux Rélation de Commutation. Paris: Armand Colin 1968.

11. Johansen, S.: Z. Wahrscheinlichkeitstheorie verw. Geb. 5, 304 (1966).

R. F. Streater

Mathematics Department

Beford College, Regents Park

London, N.W. 1, England 